\title{
Productivity Formulae of an Infinite-Conductivity Hydraulically Fractured Well Producing at Constant Wellbore Pressure Based on Numerical Solutions of a Weakly Singular Integral Equation of the First Kind
}

\author{
Chaolang $\mathrm{Hu}^{1}{ }^{1}$ Jing $\mathrm{Lu}^{2}{ }^{2}$ and Xiaoming $\mathrm{He}^{3}$ \\ ${ }^{1}$ College of Mathematics, Sichuan University, Sichuan, Chengdu 610041, China \\ ${ }^{2}$ Department of Petroleum Engineering, The Petroleum Institute, P.O. Box 2533, Abu Dhabi, UAE \\ ${ }^{3}$ Department of Mathematics and Statistics, Missouri University of Science and Technology, Rolla, \\ MO 65401, USA
}

Correspondence should be addressed to Xiaoming He, hex@mst.edu

Received 13 April 2012; Accepted 29 May 2012

Academic Editor: Kue-Hong Chen

Copyright (C) 2012 Chaolang Hu et al. This is an open access article distributed under the Creative Commons Attribution License, which permits unrestricted use, distribution, and reproduction in any medium, provided the original work is properly cited.

In order to increase productivity, it is important to study the performance of a hydraulically fractured well producing at constant wellbore pressure. This paper constructs a new productivity formula, which is obtained by solving a weakly singular integral equation of the first kind, for an infinite-conductivity hydraulically fractured well producing at constant pressure. And the two key components of this paper are a weakly singular integral equation of the first kind and a steady-state productivity formula. A new midrectangle algorithm and a Galerkin method are presented in order to solve the weakly singular integral equation. The numerical results of these two methods are in accordance with each other. And then the solutions of the weakly singular integral equation are utilized for the productivity formula of hydraulic fractured wells producing at constant pressure, which provide fast analytical tools to evaluate production performance of infinite-conductivity fractured wells. The paper also shows equipotential threads, which are generated from the numerical results, with different fluid potential values. These threads can be approximately taken as a family of ellipses whose focuses are the two endpoints of the fracture, which is in accordance with the regular assumption in Kuchuk and Brigham, 1979.

\section{Introduction}

The main objective of hydraulic fracturing for well stimulation is to increase well productivity by creating a highly conductive path some distance away from the skin zone into the 
formation. The fracture creates more surface area to the wellbore without drilling another well. Since more reservoir area is in direct communication with the wellbore, a greater volume of fluid can be produced into the wellbore per unit time, resulting in an increased production rate. This basic objective has not changed since hydraulic fracturing was introduced in the early 1950s. At the beginning, hydraulic fracturing was considered a good mean to increase productivity of the wells completed in low-permeability reservoirs. Now, it has become an integral part of most well completions.

For hydraulically fractured wells, unsteady-state pressure transient testings are useful tools for evaluating insitu reservoir and wellbore parameters that describe the production characteristics. The use of transient well testing for determining reservoir parameters and productivity of fractured wells has become common, and determination of transient pressure behavior and productivity for fractured wells has aroused considerable interest over the past decades.

Numerous analytic solutions have been presented for the pressure behavior of a fractured well producing at constant flow rate. An extensive literature survey on fractured wells producing at constant flow rates can be found. Guppy et al. present for the first time a technique that analyzes buildup and drawdown data from wells produced at constant pressure with turbulent flow in the fracture [1]. Cinco-Ley and Samaniego-V studied the early-time pressure data for a well intercepted by a finite-conductivity vertical fracture [2]. Ozkan et al. described the characteristics of a well producing at a constant pressure in a natural fractured reservoir [3]. Guppy and Cinco-Ley presented semianalytic solutions for unsteady-state flow behavior of a well intersecting a vertical fracture and analytic solutions for defining certain portions of the early time data for various types of fracture conductivity [4]. Nashawi discussed a semianalytical equation which incorporates the effects of non-Darcy flow in the fracture [5]. Kuchuk and Brigham presented analytical solutions to elliptical flow problems that are applicable to infinite-conductivity vertically fractured wells, elliptically shaped reservoirs, and anisotropic reservoirs producing at constant rate or pressure [6].

Although most well test analysis methods for hydraulically fractured wells assume constant rate production, it must be pointed out that the constant rate production is difficult to maintain, and constant wellbore pressure production conditions are not uncommon. Examples of conditions under which constant pressure is maintained at a well include production into a constant pressure separator or pipeline, open flow to the atmosphere, or production from a low permeability reservoir. It is important to consider wells producing at constant pressure rather than constant rate during large portions of the production life of tight reservoirs [4].

This paper proposes a new productivity model of an infinite-conductive hydraulically fractured well producing at constant pressure. The weakly singular integral equation [7-9] arising from this model has attracted much attention for its numerical solutions [10-12].

In order to solve the problem of constant wellbore pressure production, the numerical solution to a weakly singular integral equation is required, and a new midrectangular algorithm is used to solve the weakly singular integral equation; the corresponding numerical results are compared with those by the Galerkin method.

Furthermore, the solutions of the weakly singular integral equation are used in the productivity formulae of hydraulically fractured wells producing at constant pressure. These formulae provide fast analytical tools to evaluate production performance of infiniteconductivity fractured wells. And equipotential threads with different fluid potential values, which can be approximately taken as a family of ellipses whose focuses are the two endpoints of the fracture, are also shown. 


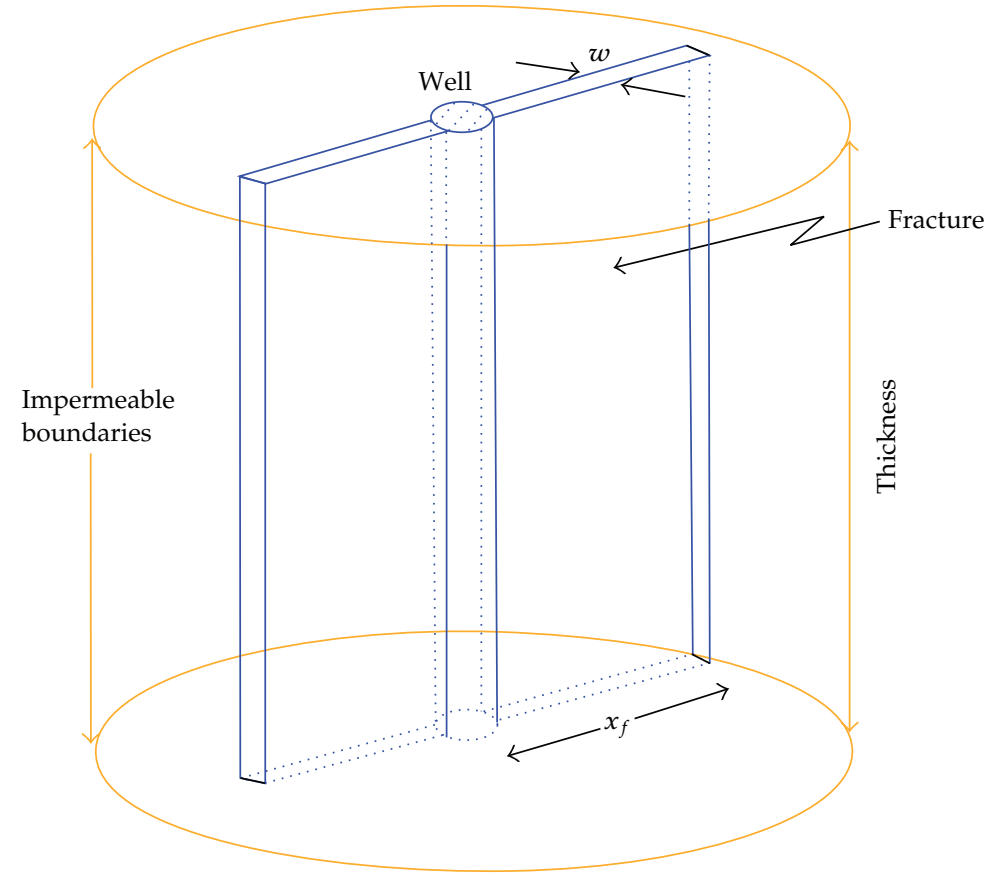

Figure 1: Schematic of ideal fracture.

The rest of this paper is organized as follows. In Section 2, the new model is derived. In Section 3, the Galerkin method and the corresponding numerical results are presented. In Section 4, the midrectangle algorithm is constructed, and the corresponding numerical experiments are presented. Then conclusions are reached in Section 5.

\section{Infinite-Conductivity Hydraulically Fractured Well Model Producing at Constant Wellbore Pressure}

In this section, we will construct a new productivity model of an infinite-conductivity hydraulically fractured well producing at constant wellbore pressure.

Figure 1 represents an ideal vertical fracture. The usual assumptions apply; that is, the porous medium is isotropic, horizontal, homogeneous, and uniform in thickness and has constant permeability. Also, the fracture fully penetrates the vertical extent of the formation and is the same length on both sides of the well.

The porous media domain is as follows:

$$
\Omega=\left\{(x, y, z) \mid x^{2}+y^{2}<R_{e}^{2}, 0<z<H\right\}
$$

where $R_{e}$ is cylinder radius and $\Omega$ is the cylindrical body. Since both the vertical well and the hydraulic fracture are fully penetrating, we may use two-dimensional model to study the pressure behavior. Assume the fracture length is $L, L=2 x_{f}$, the fracture is taken as a line sink in the two-dimensional space, and the coordinates of the two ends are $\left(-x_{f}, 0\right)$ and $\left(\mathrm{x}_{f}, 0\right)$. Supposing that point $\left(x^{\prime}, 0\right)$ is on the fracture, in order to obtain the pressure at point 
$(x, y)$ caused by the point $\left(x^{\prime}, 0\right)$, we have to obtain the basic solution of the following partial differential equation in $\Omega$ :

$$
\frac{K}{\mu} \frac{\partial^{2} P}{\partial x^{2}}+\frac{K}{\mu} \frac{\partial^{2} P}{\partial y^{2}}=-q\left(x^{\prime}\right) \delta\left(x-x^{\prime}\right) \delta(y)
$$

where $P(x, y)$ is the pressure at point $(x, y) . K$ is the formation permeability, $\mu$ is viscosity, $q\left(x^{\prime}\right)$ is the flow rate at point $\left(x^{\prime}, 0\right), \delta(y), \delta\left(x-x^{\prime}\right)$ are Dirac functions and

$$
x^{\prime} \in\left[-x_{f}, x_{f}\right]
$$

that is, the hydraulic fracture is between $\left[-x_{f}, x_{f}\right]$.

Let

$$
\left.P\right|_{r \rightarrow \infty}=P_{i}>0,
$$

where $\left.P\right|_{r \rightarrow \infty}$ is the pressure at infinite distance away from the fracture. Because the hydraulically fractured well is infinite conductivity, the pressure of the fracture denoted by $P_{0}$ is uniform. That is

$$
P(x, 0)=P_{0}=\text { constant }, \quad \forall x \in\left[-x_{f}, x_{f}\right] .
$$

Thus, if $q\left(x^{\prime}\right)$ is known, the total productivity is given by

$$
Q=\int_{0}^{H} \int_{-x_{f}}^{x_{f}} q\left(x^{\prime}\right) d x^{\prime} d z=H \int_{-x_{f}}^{x_{f}} q\left(x^{\prime}\right) d x^{\prime} .
$$

In order to simplify the previously mentioned equations, we take the following dimensionless transforms:

$$
\begin{array}{cc}
x_{D}=\frac{x}{2 x_{f}}, & y_{D}=\frac{y}{2 x_{f}}, \\
P_{D}=\frac{P_{i}-P}{P_{i}}, & P_{D 0}=\frac{P_{i}-P_{0}}{P_{i}} .
\end{array}
$$

Obviously, we obtain

$$
\begin{gathered}
\frac{\partial x_{D}}{\partial x}=\frac{1}{2 x_{f}} \\
\frac{\partial P}{\partial x}=\left(\frac{\partial P}{\partial x_{D}}\right)\left(\frac{\partial x_{D}}{\partial x}\right)=\frac{1}{2 x_{f}} \frac{\partial P}{\partial x_{D}} \\
\frac{\partial^{2} P}{\partial x^{2}}=\frac{\partial}{\partial x}\left(\frac{\partial P}{\partial x}\right)=\frac{1}{4 x_{f}^{2}} \frac{\partial^{2} P}{\partial x_{D}^{2}}
\end{gathered}
$$


Since

$$
P_{D}=\frac{P_{i}-P}{P_{i}}
$$

then

$$
\frac{\partial^{2} P}{\partial x^{2}}=-\frac{P_{i}}{4 x_{f}^{2}} \frac{\partial^{2} P_{D}}{\partial x_{D}^{2}}
$$

In the same manner, we have

$$
\begin{aligned}
\frac{\partial^{2} P}{\partial y^{2}} & =-\frac{P_{i}}{4 x_{f}^{2}} \frac{\partial^{2} P_{D}}{\partial y_{D}^{2}} \\
\delta\left(x-x^{\prime}\right) \delta(y) & =\delta\left[2 x_{f}\left(x_{D}-x_{D}^{\prime}\right)\right] \delta\left(2 x_{f} y_{D}\right) \\
& =\left(2 x_{f}\right)^{-2} \delta\left(x_{D}-x_{D}^{\prime}\right) \delta\left(y_{D}\right) .
\end{aligned}
$$

So

$$
\begin{aligned}
\frac{K}{\mu} \frac{\partial^{2} P}{\partial x^{2}}+\frac{K}{\mu} \frac{\partial^{2} P}{\partial y^{2}} & =-\frac{K}{\mu} \frac{P_{i}}{4 x_{f}^{2}}\left(\frac{\partial^{2} P_{D}}{\partial x_{D}^{2}}+\frac{\partial^{2} P_{D}}{\partial y_{D}^{2}}\right) \\
& =-q\left(x_{f} x_{D}^{\prime}\right)\left(2 x_{f}\right)^{-2} \delta\left(x_{D}-x_{D}^{\prime}\right) \delta\left(y_{D}\right)
\end{aligned}
$$

That is,

$$
\frac{\partial^{2} P_{D}}{\partial x_{D}^{2}}+\frac{\partial^{2} P_{D}}{\partial y_{D}^{2}}=q_{D}\left(x_{D}^{\prime}\right) \delta\left(x_{D}-x_{D}^{\prime}\right) \delta\left(y_{D}\right)
$$

where

$$
q_{D}\left(x_{D}^{\prime}\right)=\frac{q\left(2 x_{f} x_{D}^{\prime}\right) \mu}{K P_{i}}, \quad x_{D}^{\prime} \in\left[-\frac{1}{2}, \frac{1}{2}\right]
$$

Note that after taking the dimensionless transforms, producing section $\left[-x_{f}, x_{f}\right]$ of the fractured well is changed to $[-1 / 2,1 / 2]$, and the initial conditions are changed as follows:

$$
\begin{gathered}
\left.P_{D}\right|_{r_{D} \rightarrow \infty} \longrightarrow 0^{+}, \\
q_{D}\left(x_{D}^{\prime}\right)=0, \quad x_{D}^{\prime} \notin\left[-\frac{1}{2}, \frac{1}{2}\right] .
\end{gathered}
$$


It is well known that the fundamental solution of two-dimensional Laplace equation is as follows $[13,14]$ :

$$
g\left(x, y ; x^{\prime}, y^{\prime}\right)=-\frac{\ln \left[\left(x-x^{\prime}\right)^{2}+\left(y-y^{\prime}\right)^{2}\right]^{1 / 2}}{2 \pi}
$$

Thus, if $q_{D}\left(x_{D}^{\prime}\right)$ is known, the solution to (2.13) is given by

$$
P_{D}\left(x_{D}, y_{D}\right)=\int_{-1 / 2}^{1 / 2} g\left(x_{D}, y_{D} ; x_{D}^{\prime}, 0\right) q_{D}\left(x_{D}^{\prime}\right) d x_{D}^{\prime}
$$

If the hydraulically fractured well is infinite conductivity, then we have

$$
P_{D}\left(x_{D}, 0\right)=P_{D 0}, \quad x_{D} \in\left[-\frac{1}{2}, \frac{1}{2}\right]
$$

where $P_{D 0}$ is a constant.

From (2.17), we can obtain the following equation:

$$
\int_{-1 / 2}^{1 / 2} g\left(x_{D}, 0 ; x_{D}^{\prime}, 0\right) q_{D}\left(x_{D}^{\prime}\right) d x_{D}^{\prime}=P_{D 0}
$$

and it can be simplified as follows:

$$
\left(\frac{-1}{2 \pi}\right) \int_{-1 / 2}^{1 / 2} \ln \left|x_{D}-x_{D}^{\prime}\right| q_{D}\left(x_{D}^{\prime}\right) d x_{D}^{\prime}=P_{D 0} .
$$

Let

$$
u\left(x_{D}^{\prime}\right)=\frac{q_{D}\left(x_{D}^{\prime}\right)}{2 \pi P_{D 0}}, \quad x_{D}^{\prime} \in\left[-\frac{1}{2}, \frac{1}{2}\right]
$$

Consequently, we obtain the following first kind weakly singular integral equation:

$$
\int_{-1 / 2}^{1 / 2} \ln |x-y| u(x) d x=-1, \quad \forall y \in\left[-\frac{1}{2}, \frac{1}{2}\right]
$$

Once $u(x)$ is obtained from the previous equation, we can use it to compute

$$
q_{D}(x)=2 \pi P_{D 0} u(x), \quad x \in\left[-\frac{1}{2}, \frac{1}{2}\right]
$$


Table 1: Galerkin approximations of (2.22) with $h=1 / 32$.

\begin{tabular}{lcccc}
\hline$i$ & 1 & 2 & 3 & 4 \\
\hline$c_{i}$ & 2.6610334017 & 0.9490861410 & 0.8841883020 & 0.7384058892 \\
$c_{i+4}$ & 0.6653019950 & 0.6118426439 & 0.5732934677 & 0.5440894309 \\
$c_{i+8}$ & 0.5215521538 & 0.5039599049 & 0.4902132354 & 0.4795754716 \\
$c_{i+12}$ & 0.4715448802 & 0.4657790288 & 0.4620502406 & 0.4602190183 \\
$c_{i+16}$ & 0.4602190183 & 0.4620502406 & 0.4657790288 & 0.4715448802 \\
$c_{i+20}$ & 0.4795754716 & 0.4902132354 & 0.5039599049 & 0.5215521538 \\
$c_{i+24}$ & 0.5440894309 & 0.5732934677 & 0.6118426439 & 0.6653019950 \\
$c_{i+28}$ & 0.7384058892 & 0.8841883020 & 0.9490861410 & 2.6610334017 \\
\hline
\end{tabular}

Using

$$
q_{D}\left(x_{D}^{\prime}\right)=\frac{q\left(2 x_{f} x_{D}^{\prime}\right) \mu}{K P_{i}}, \quad x_{D}^{\prime} \in\left[-\frac{1}{2}, \frac{1}{2}\right]
$$

we can obtain

$$
q\left(2 x_{f} x_{D}^{\prime}\right)=\frac{K P_{i}}{\mu} q_{D}\left(x_{D}^{\prime}\right), \quad x_{D}^{\prime} \in\left[-\frac{1}{2}, \frac{1}{2}\right]
$$

The total productivity of the hydraulically fractured well is as follows:

$$
\begin{aligned}
Q & =H \int_{-x_{f}}^{x_{f}} q\left(x^{\prime}\right) d x^{\prime} \\
& =2 x_{f} H \int_{-1 / 2}^{1 / 2} q\left(2 x_{f} x_{D}^{\prime}\right) d x_{D}^{\prime} \\
& =2 x_{f}\left(\frac{K P_{i} H}{\mu}\right) \int_{-1 / 2}^{1 / 2} q_{D}\left(x_{D}^{\prime}\right) d x_{D}^{\prime} \\
& =4 x_{f} \pi P_{D 0}\left(\frac{K P_{i} H}{\mu}\right) \int_{-1 / 2}^{1 / 2} u(x) d x \\
& =4 x_{f} \pi\left(\frac{P_{i}-P_{0}}{P_{i}}\right)\left(\frac{K P_{i} H}{\mu}\right) \int_{-1 / 2}^{1 / 2} u(x) d x, \\
Q & =4 x_{f} \pi \frac{\left(P_{i}-P_{0}\right) K H}{\mu} \int_{-1 / 2}^{1 / 2} u(x) d x .
\end{aligned}
$$

Remark 2.1. Equation (2.22) is the first kind weakly singular integral equation, which can be solved with the numerical methods introduced in Sections 3 and 4. And the total productivity can be obtained by (2.26). 
Table 2: Galerkin approximations of (3.11) with different $h$.

\begin{tabular}{lcccc}
\hline$i$ & 1 & 2 & 3 & 4 \\
\hline$Q\left(1 / 2^{i}\right)$ & & 0.6932919850 & 0.7068397093 & 0.7139761064 \\
$Q\left(1 / 2^{i+4}\right)$ & 0.7176334503 & 0.7194835363 & 0.7204138104 & 0.7208802386 \\
$Q\left(1 / 2^{i+8}\right)$ & 0.7211137731 & 0.7212306202 & 0.7212890637 & 0.7213182904 \\
\hline
\end{tabular}

Table 3: Mid-rectangle approximations of (2.22) with $h=1 / 32$.

\begin{tabular}{lcccc}
\hline$i$ & 1 & 2 & 3 & 4 \\
\hline$c_{i}$ & 2.4652280029 & 1.1168311123 & 0.8795040869 & 0.7498254718 \\
$c_{i+4}$ & 0.6700249966 & 0.6154959679 & 0.5759489664 & 0.5461808975 \\
$c_{i+8}$ & 0.5215521538 & 0.5039599049 & 0.4902132354 & 0.4807028032 \\
$c_{i+12}$ & 0.4725784047 & 0.4667483804 & 0.462979470 & 0.4611289341 \\
$c_{i+16}$ & 0.4611289341 & 0.462979470 & 0.4667483804 & 0.4725784047 \\
$c_{i+20}$ & 0.4807028032 & 0.4902132354 & 0.5039599049 & 0.5215521538 \\
$c_{i+24}$ & 0.5440894309 & 0.5732934677 & 0.6154959679 & 0.6700249966 \\
$c_{i+28}$ & 0.7498254718 & 0.8795040869 & 1.1168311123 & 2.4652280029 \\
\hline
\end{tabular}

Remark 2.2. From Tables 1 and 2 in Section 3 or Tables 3 and 4 in Section 4 , we can find that $\int_{-1 / 2}^{1 / 2} u(x) d x$ is a constant. So the total productivity formula is as follows:

$$
\begin{aligned}
Q & =4 x_{f} \pi \frac{\left(P_{i}-P_{0}\right) K H}{\mu} \int_{-1 / 2}^{1 / 2} u(x) d x \\
& \approx 4 x_{f} \pi \frac{\left(P_{i}-P_{0}\right) K H}{\mu} h \sum_{j=0}^{n-1} u_{j} \\
& \approx 4 x_{f} \pi \frac{\left(P_{i}-P_{0}\right) K H}{\mu} 0.7213 \\
& =9.0641 x_{f} \frac{\left(P_{i}-P_{0}\right) K H}{\mu} \\
& =4.53205 \frac{\left(P_{i}-P_{0}\right) K H L}{\mu},
\end{aligned}
$$

where $L$ is the length of the hydraulically fractured well.

\section{Galerkin Method for Steady-State Productivity Computation of the Hydraulically Fractured Well}

In this section, we will present the Galerkin method, which has a mature analysis framework, to solve the weakly singular integral equation (2.22). Once $u(x)$ of $(2.22)$ is known, the approximation of (2.26) is also presented. 
Table 4: Mid-rectangle approximations of (4.16) with different $h$.

\begin{tabular}{lcccc}
\hline$i$ & 1 & 2 & 3 & 4 \\
\hline$Q^{\prime}\left(1 / 2^{i}\right)$ & & 0.6859163596 & 0.7044679397 & 0.7134577603 \\
$Q^{\prime}\left(1 / 2^{i+4}\right)$ & 0.7177072748 & 0.7196865358 & 0.7205981981 & 0.7210138248 \\
$Q^{\prime}\left(1 / 2^{i+8}\right)$ & 0.7212012467 & 0.7212846926 & 0.7213212664 & 0.7213369747 \\
\hline
\end{tabular}

Let

$$
\begin{gathered}
h=\frac{1}{n}, \quad x_{j}=-\frac{1}{2}+j h, \quad(j=0, \ldots, n), \\
\Gamma=\left[-\frac{1}{2}, \frac{1}{2}\right], \quad \Gamma_{j}=\left[x_{j-1}, x_{j}\right], \quad(j=1, \ldots, n) .
\end{gathered}
$$

Clearly,

$$
\Gamma=\bigcup_{j=1}^{n} \Gamma_{j}
$$

Let

$$
\begin{gathered}
e_{j}(x)= \begin{cases}1, & x \in \Gamma_{j}, \\
0, & x \notin \Gamma_{j,}\end{cases} \\
S^{h}=\operatorname{span}\left\{e_{j}(x), j=1, \ldots, n\right\} .
\end{gathered}
$$

Define the energy inner product

$$
\begin{aligned}
{[u, v] } & =(K u, v) \\
& =-\iint_{\Gamma} \ln |x-y| u(x) v(y) d s_{x} d s_{y}, \quad \forall u, v \in L_{2}(\Gamma)
\end{aligned}
$$

and the energy norm

$$
\|u\|^{2}=[u, u] .
$$

The weak formulation is to find $u \in L_{2}(\Gamma)$ such that

$$
[u, v]=(g, v), \quad \forall v \in L_{2}(\Gamma)
$$

And the Galerkin formulation is to find

$$
u^{h}(x)=\sum_{j=1}^{n} c_{j} e_{j}(x) \in S^{h}
$$


such that

$$
\left[u^{h}, v^{h}\right]=\left(g, v^{h}\right), \quad \forall v^{h} \in S^{h} .
$$

Then the matrix representation of the approximation is

$$
A \bar{c}=\bar{g},
$$

where

$$
\begin{gathered}
A=\left(a_{i j}\right)_{n \times n^{\prime}} \\
a_{i j}=-\int_{\Gamma_{i}} \int_{\Gamma_{j}} \ln |x-y| d s_{x} d s_{y}, \quad(i, j=1, \ldots, n), \\
\bar{c}=\left[c_{1}, c_{2}, \ldots, c_{n}\right]^{T}, \\
\bar{g}=\left[g_{1}, g_{2}, \ldots, g_{n}\right]^{T}, \\
g_{j}=\left(g, e_{j}\right)=\iint_{\Gamma} g(x) e_{j}(y) d s_{x} d s_{y}, \quad(j=1, \ldots, n) .
\end{gathered}
$$

Once the solution of (3.9) is obtained, we can utilize it to obtain the total productivity of the hydraulically fractured well by the following formula:

$$
Q(h)=h \sum_{j=1}^{n} c_{j}
$$

In fact

$$
\begin{aligned}
Q(h) & =\int_{-1 / 2}^{1 / 2} u^{h}(x) d x \\
& =\int_{-1 / 2}^{1 / 2} \sum_{j=1}^{n} c_{j} e_{j}(x) d x \\
& =\sum_{j=1}^{n} c_{j} \int_{-1 / 2}^{1 / 2} e_{j}(x) d x \\
& =\sum_{j=1}^{n} c_{j} h,
\end{aligned}
$$

which completes the proof of Formula (3.11).

In the following, we will present the error estimate of the Galerkin approximation. Since $S^{h} \subset L^{2}(\Gamma)$, then (3.6) and (3.8) lead to

$$
\left[u^{h}-u, v\right]=0, \quad \forall v \in S^{h}
$$


Hence

$$
u^{h}=P^{h} u,
$$

where $P^{h}: L_{2}(\Gamma) \mapsto S^{h}$ is the orthogonal projection with respect to the energy inner product. Consequently, there holds

$$
\left\|u^{h}-u\right\|=\inf _{v \in S^{h}}\|u-v\|
$$

Using (3.15), the following theorem, which is recalled from $[7,8]$, is valid.

Theorem 3.1 (see $[7,8]$ ). If $\Gamma$ is sufficiently smooth, $g$ is a smooth function on $\Gamma$, and $S^{h}$ is a subspace which is construct of piecewise smooth constant functions, then there is a constant $c>0$ such that

$$
\begin{gathered}
\left\|u^{h}-u\right\| \leq c h^{2 / 3}, \\
\left|\left(u^{h}-u, g\right)\right| \leq c h^{3} .
\end{gathered}
$$

Now we turn to the implementation issue of (3.9). Clearly,

$$
\lim _{h \rightarrow 0} h \ln h=0 .
$$

Using (3.17), we can obtain

$$
\begin{aligned}
a_{i j}= & -\int_{\Gamma_{i}} \int_{\Gamma_{j}} \ln |x-y| d s_{x} d s_{y} \\
= & \frac{1}{2}(j-i-1)^{2} h^{2} \ln |(j-i-1) h|-(j-i)^{2} h^{2} \ln |(j-i) h| \\
& -\frac{3}{2} h^{2}+\frac{1}{2}(j-i+1)^{2} h^{2} \ln |(j-i+1) h|, \\
g_{j}= & \left(g, e_{j}\right) \\
= & \iint_{\Gamma} g(x) e_{j}(y) d s_{x} d s_{y}
\end{aligned}
$$




$$
\begin{aligned}
& =\int_{\Gamma} g(x) d s_{x} \int_{\Gamma} e_{j}(y) d s_{y} \\
& =\int_{\Gamma} g(x) d s_{x} h .
\end{aligned}
$$

In the numerical experiment, we choose $g(x)=-1$ and $\Gamma=[-1 / 2,1 / 2]$ in the previous algorithm in order to obtain the numerical solutions of (2.22). Table 1 shows the Galerkin approximations with $h=1 / 32$, from which we can see that the solution is symmetric, that is, $c_{i}=c_{\mathrm{n}+1-i}, i=1, \ldots, n$. And Table 2 shows the total productivity of fractured well with different $h$, which is convergent when $h \rightarrow 0$. More observations and conclusions from the numerical results will be presented at the end of Section 4 .

\section{Midrectangle Algorithm for Steady-State Productivity Computation of the Hydraulically Fractured Well}

In this section, we will construct and analyze a midrectangle algorithm, which is much more convenient to implement but still has similar accuracy to that of the Galerkin method.

We first prove the following theorem, which will lead to the algorithm and provide the error estimate of the method.

Theorem 4.1. For any $g(x) \in C_{[t-a, t+a]}^{2}$, there holds

$$
\int_{t-a}^{t+a} g(x) \ln |x-t| d x=2 g(t) a(\ln a-1)+O\left(a^{3} \ln a\right) .
$$

Proof. Let

$$
h=\frac{a}{n}, \quad x_{i}=t-a+i h, \quad(i=0,1, \ldots, n-1, n, n+1, \ldots, 2 n) .
$$

Obviously,

$$
x_{n}=t, \quad x_{i}+x_{2 n-i}=2 t .
$$

By Taylor expansion, we can obtain

$$
g\left(x_{i}\right)+g\left(x_{2 n-i}\right)=2 g(t)+\frac{g^{\prime \prime}\left(\zeta_{i}\right)}{2}\left(x_{i}-t\right)^{2} .
$$

That is,

$$
g\left(x_{i}\right)+g\left(x_{2 n-i}\right)=2 g(t)+\frac{g^{\prime \prime}\left(\zeta_{i}\right)}{2}((n-i) h)^{2} .
$$


Hence

$$
\begin{aligned}
& \int_{t-a}^{t+a} g(x) \ln |x-t| d x=\lim _{h \rightarrow 0}\left[h \sum_{i=0}^{n-1} g\left(x_{i}\right) \ln \left|x_{i}-t\right|+h \sum_{i=n+1}^{2 n} g\left(x_{i}\right) \ln \left|x_{i}-t\right|\right] \\
& =\lim _{h \rightarrow 0}\left[h \sum_{i=0}^{n-1} g\left(x_{i}\right) \ln (a-i h)+h \sum_{i=n+1}^{2 n} g\left(x_{i}\right) \ln (i h-a)\right] \\
& =\lim _{h \rightarrow 0}\left[h \sum_{i=0}^{n-1} g\left(x_{i}\right) \ln (a-i h)+h \sum_{i=n+1-2 n}^{2 n-2 n} g\left(x_{i+2 n}\right) \ln ((i+2 n) h-a)\right] \\
& =\lim _{h \rightarrow 0}\left[h \sum_{i=0}^{n-1} g\left(x_{i}\right) \ln (a-i h)+h \sum_{i=1-n}^{0} g\left(x_{i+2 n}\right) \ln ((i+2 n) h-a)\right] \\
& =\lim _{h \rightarrow 0}\left[h \sum_{i=0}^{n-1} g\left(x_{i}\right) \ln (a-i h)+h \sum_{i=-(1-n)}^{-0} g\left(x_{-i+2 n}\right) \ln ((-i+2 n) h-a)\right] \\
& =\lim _{h \rightarrow 0}\left[h \sum_{i=0}^{n-1} g\left(x_{i}\right) \ln (a-i h)+h \sum_{i=n-1}^{0} g\left(x_{-i+2 n}\right) \ln ((-i+2 n) h-a)\right] \\
& =\lim _{h \rightarrow 0}\left[h \sum_{i=0}^{n-1} g\left(x_{i}\right) \ln (a-i h)+h \sum_{i=0}^{n-1} g\left(x_{-i+2 n}\right) \ln ((-i+2 n) h-a)\right] \\
& =\lim _{h \rightarrow 0}\left[h \sum_{i=0}^{n-1} g\left(x_{i}\right) \ln (a-i h)+h \sum_{i=0}^{n-1} g\left(x_{2 n-i}\right) \ln (-i h+2 n h-a)\right] \\
& =\lim _{h \rightarrow 0}\left[h \sum_{i=0}^{n-1} g\left(x_{i}\right) \ln (a-i h)+h \sum_{i=0}^{n-1} g\left(x_{2 n-i}\right) \ln (a-i h)\right] \\
& =\lim _{h \rightarrow 0}\left[h \sum_{i=0}^{h-1}\left(g\left(x_{i}\right)+g\left(x_{2 n-i}\right)\right) \ln (a-i h)\right] \\
& \left.=\lim _{h \rightarrow 0}\left[h \sum_{i=0}^{n-1}\left(2 g(t)+\frac{g^{\prime \prime}\left(\zeta_{i}\right)}{2}((n-i) h)^{2}\right)\right) \ln (a-i h)\right] \\
& =\lim _{h \rightarrow 0}\left[h \sum_{i=0}^{n-1}(2 g(t) \ln (a-i h))\right]+\lim _{h \rightarrow 0}\left[h \sum_{i=0}^{n-1} \frac{g^{\prime \prime}\left(\zeta_{i}\right)}{2}((n-i) h)^{2} \ln (a-i h)\right] \\
& =\lim _{h \rightarrow 0}\left[h \sum_{i=0}^{n-1}(2 g(t) \ln ((n-i) h)]+\lim _{h \rightarrow 0}\left[h \sum_{i=0}^{n-1} \frac{g^{\prime \prime}\left(\zeta_{i}\right)}{2}((n-i) h)^{2} \ln ((n-i) h)\right]\right. \\
& =I_{1}+I_{2} \text {. }
\end{aligned}
$$


Here

$$
\begin{aligned}
I_{1} & =\lim _{h \rightarrow 0}\left[h \sum_{i=0}^{n-1}(2 g(t) \ln ((n-i) h)]\right. \\
& =2 g(t) \lim _{h \rightarrow 0}\left[h \sum_{i=0}^{n-1}(\ln ((n-i) h)]\right. \\
& =2 g(t) \lim _{h \rightarrow 0}\left[h \sum_{i=1}^{n} \ln (i h)\right] \\
& =2 g(t) \int_{0}^{a} \ln x d x \\
& =\left.2 g(t)[x \ln x-x]\right|_{0} ^{a}=2 g(t)[a \ln a-a],
\end{aligned}
$$

where we have used the result of $\lim _{x \rightarrow 0} x \ln x=0$. Now we consider $I_{2}$. Let $m \leq g^{\prime \prime}\left(\zeta_{i}\right) \leq M,(i=$ $0,1, \ldots, n-1)$. There exists such a constant $k$ that

$$
\begin{aligned}
I_{2} & =\lim _{h \rightarrow 0}\left[h \sum_{i=0}^{n-1} \frac{g^{\prime \prime}\left(\zeta_{i}\right)}{2}((n-i) h)^{2} \ln ((n-i) h)\right] \\
& =\frac{k}{2} \lim _{h \rightarrow 0}\left[h \sum_{i=0}^{n-1}((n-i) h)^{2} \ln ((n-i) h)\right] \\
& =\frac{k}{2} \lim _{h \rightarrow 0}\left[h \sum_{i=1}^{n}(i h)^{2} \ln (i h)\right] \\
& =\frac{k}{2} \int_{0}^{a} x^{2} \ln x d x \\
& =\left.\frac{k}{2}\left(\frac{x^{3}}{3} \ln x-\frac{x^{3}}{9}\right)\right|_{0} ^{a} \\
& =\frac{k}{2}\left(\frac{a^{3}}{3} \ln a-\frac{a^{3}}{9}\right) .
\end{aligned}
$$

Plugging $I_{1}$ and $I_{2}$ into (4.6), the proof is completed.

Let

$$
\begin{aligned}
& h=\frac{1}{n}, \quad x_{j}=-\frac{1}{2}+j h, \quad(j=0,1, \ldots, n), \\
& y_{j}=-\frac{1}{2}+\left(j+\frac{1}{2}\right) h, \quad(j=0,1, \ldots, n-1) .
\end{aligned}
$$


Clearly,

$$
y_{j}=\frac{1}{2}\left(x_{j}+x_{j+1}\right), \quad(j=0,1, \ldots, n-1)
$$

Let $u_{j}$ be the approximation of $u\left(y_{j}\right)$, and let $y_{i}=(1 / 2)\left(x_{i}+x_{i+1}\right),(i=0,1, \ldots, n-1)$ be the singular points in (2.22). Then

$$
\begin{aligned}
\int_{-1 / 2}^{1 / 2} u(x) \ln \left|x-y_{i}\right| d x= & \sum_{j=0}^{n-1} \int_{x_{j}}^{x_{j+1}} u(x) \ln \left|x-y_{i}\right| d x \\
= & \sum_{j=0}^{i-1} \int_{x_{j}}^{x_{j+1}} u(x) \ln \left|x-y_{i}\right| d x+\int_{x_{i}}^{x_{i+1}} u(x) \ln \left|x-y_{i}\right| d x \\
& +\sum_{j=i+1}^{n-1} \int_{x_{j}}^{x_{j+1}} u(x) \ln \left|x-y_{i}\right| d x \\
= & \sum_{j=0}^{i-1} h\left[u\left(y_{j}\right) \ln \left|y_{j}-y_{i}\right|+O\left(h^{2}\right)\right]+2 u\left(y_{i}\right) \frac{h}{2}\left(\ln \frac{h}{2}-1\right) \\
& +O\left(\left(\frac{h}{2}\right)^{3} \ln \frac{h}{2}\right)+\sum_{j=i+1}^{n-1} h\left[u\left(y_{j}\right) \ln \left|y_{j}-y_{i}\right|+O\left(h^{2}\right)\right] \\
= & \sum_{j=0, j \neq i}^{n-1} h u\left(y_{j}\right) \ln \left|y_{j}-y_{i}\right|+h u\left(y_{i}\right) \ln \frac{h}{2 e}+O\left(h^{2}\right) .
\end{aligned}
$$

Hence we obtain the following theorem.

Theorem 4.2. For all $u(x) \in C_{[-1 / 2,1 / 2]}^{\prime \prime}, h=1 / n, x_{j}=(-1 / 2)+j h, j=0,1, \ldots, n, y_{j}=-1 / 2+$ $(j+1 / 2) h,(j=0,1, \ldots, n-1)$, there holds

$$
\begin{aligned}
\int_{-1 / 2}^{1 / 2} u(x) \ln \left|x-y_{i}\right| d x= & \sum_{j=0, j \neq i}^{n-1} h u\left(y_{j}\right) \ln \left|y_{j}-y_{i}\right|+u\left(y_{i}\right) h \ln \frac{h}{2 e} \\
& +O\left(h^{2}\right), \quad(i=0,1, \ldots, n-1) .
\end{aligned}
$$

From Theorem 4.2, we can construct the following midrectangle algorithm for (2.22):

$$
\begin{aligned}
-1 & =\int_{-1 / 2}^{1 / 2} \mathrm{u}(x) \ln \left|x-y_{i}\right| d x \\
& \approx \sum_{j=0, j \neq i}^{n-1}\left(h \ln \left|y_{j}-y_{i}\right|\right) u_{j}+\left(h \ln \frac{h}{2 e}\right) u_{i}, \quad(i=0,1, \ldots, n-1) .
\end{aligned}
$$


The error estimate of this method is given by Theorem 4.2. And the matrix representation of $(4.13)$ is

$$
A \bar{u}=-I,
$$

where

$$
\begin{gathered}
\bar{u}=\left[u_{0}, u_{1}, \ldots, u_{n-1}\right]^{T}, \quad I=[1,1, \ldots, 1]^{T}, \\
A=\left(a_{i j}\right)_{i, j=0^{\prime}}^{n-1} \\
a_{i, j}=\left\{\begin{array}{cc}
h \ln \frac{h}{2 e} \quad & i=j \\
h \ln (|j-i| h) & i \neq j
\end{array}, \quad(i, j=0,1, \ldots, n-1) .\right.
\end{gathered}
$$

After $\bar{u}$ in (4.14) is obtained, the total productivity of the hydraulically fractured well can be approximated as follows:

$$
Q^{\prime}(h)=h \sum_{j=0}^{n-1} u_{j}
$$

Table 3 shows the midrectangle approximations with $h=1 / 32$. And Table 4 shows the total productivity of fractured well with different $h$. The numerical results perform similarly to those of the Galerkin method presented in Tables 1 and 2.

From Tables 1 and 3, we can find that when a hydraulically fractured well is producing at constant wellbore pressure, the point convergence intensity at endpoint is bigger than that of midpoint, and the point convergence intensity is at monotone decreasing trend from endpoint to midpoint.

From Tables 2 and 4, we can see that the numerical results converge to

$$
0.7213 \approx \int_{-1 / 2}^{1 / 2} u(x) d x \approx Q(h) \approx Q^{\prime}(h) .
$$

Furthermore, this constant is independent of well length, permeability, viscosity, and other fluid properties. Hence this result can be used to calculate flow rates for many realistic cases as follows:

$$
Q=0.7213 \times 2 \pi \times L \times \frac{K H}{\mu} \times\left(P_{i}-P_{0}\right) .
$$

Here $L$ is the length of the hydraulically fractured well, $K$ is permeability, $\mu$ is viscosity $P_{i}$ is pressure at an infinite distance from the well, and $P_{0}$ is wellbore pressure of the hydraulically fractured well. From (4.18), we can see that when a hydraulically fractured well is producing at constant wellbore pressure, its flow rate is directly proportional to well length, permeability, and pressure difference. 


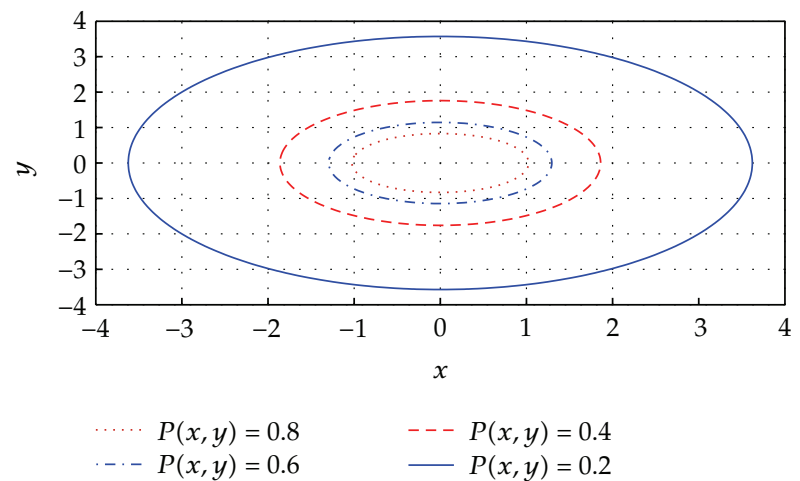

Figure 2: Profile of equipotential threads of a hydraulically fractured well.

If $\left(P_{i}-P_{0}\right)$ is a constant, the productivity index is given by

$$
J_{v}=0.7213 \times 2 \pi \times L \times \frac{K H}{\mu}=4.53205 \times \frac{K H L}{\mu} .
$$

Using (2.17) and the results in Table 1, we can calculate the pressure at a point $(x, y)$ as follows:

$$
P(x, y) \approx\left(\frac{h}{2 \pi}\right) \sum_{i=0}^{31} u_{i} \ln \left(\sqrt{\left(x-x_{i+0.5}\right)^{2}+y^{2}}\right) .
$$

And the points, which satisfy

$$
P(x, y) \approx\left(\frac{h}{2 \pi}\right) \sum_{i=0}^{31} u_{i} \ln \left(\sqrt{\left(x-x_{i+0.5}\right)^{2}+y^{2}}\right)=\text { constant }
$$

form equipotential threads when the well is producing at constant wellbore pressure. Figure 2 shows equipotential threads with different fluid potential values. It can be found that the equipotential threads can be approximately taken as a family of ellipses whose focuses are the two endpoints of the hydraulic fractured well. This conclusion is in accordance with the regular assumption in [6].

\section{Conclusions}

This paper proposes a new model using a weakly singular integral equation for the productivity of infinite-conductivity hydraulically fractured wells producing at constant wellbore pressure. A Galerkin method and a midrectangle algorithm are constructed to solve this integral equation. And their numerical results are similar to each other. Then the numerical solutions of this equation are utilized in the productivity formula, which provide fast analytical tools to evaluate production performance of infinite-conductivity fractured wells. Furthermore, the following conclusions are obtained. 
(1) The numerical results for the weakly singular integral equation are convergent. Moreover, the point convergence intensity is symmetric. And the point convergence intensity is monotonically decreasing from the endpoints to the midpoint when a hydraulically fractured well is producing at constant wellbore pressure.

(2) The numerical approximation for the productivity is convergent when $h \rightarrow 0$.

(3) The equipotential threads, which are generated from the numerical results, can be approximately taken as a family of ellipses whose focuses are the two endpoints of the hydraulically fractured well. This conclusion is in accordance with the regular assumption in [6].

\section{References}

[1] K. H. Guppy, S. Kumar, and V. D. Kagawan, "Pressure-transient analysis for fractured wells producing at constant pressure," SPE Formation Evaluation, vol. 3, no. 1, pp. 169-178, 1988.

[2] H. Cinco-Ley and F. Samaniego-V, "Transient pressure analysis for fractured wells," Society of Petroleum Engineers of AIME, vol. 33, no. 9, pp. 1749-1766, 1981.

[3] E. Ozkan, U. Ohaeri, and R. Raghavan, "Unsteady flow to a well produced at a constant pressure in a fractured reservoir," SPE Formation Evaluation, vol. 2, no. 2, pp. 186-200, 1987.

[4] K. H. Guppy and H. Cinco-Ley, "Transient flow behavior of a vertically fractured well producing at constant pressure," Society of Petroleum Engineers of AIME, vol. 21, no. 12, pp. 9962-9963, 1981.

[5] I. S. Nashawi, "Constant-pressure analysis of infinite-conductivity fractured gas wells influenced by non-Darcy flow effects," in Proceedings of the SPE 15th Middle East Oil and Gas Show and Conference (MEOS '07), pp. 260-271, Manama, Bahrain, March 2007.

[6] F. Kuchuk and W. F. Brigham, "Transient flow in elliptical system," Society of Petroleum Engineers of AIME, vol. 19, no. 6, pp. 401-410, 1979.

[7] I. H. Sloan and A. Spence, "The Galerkin method for integral equations of the first kind with logarithmic kernel: theory," IMA Journal of Numerical Analysis, vol. 8, no. 1, pp. 105-122, 1988.

[8] I. H. Sloan and A. Spence, "The Galerkin method for integral equations of the first kind with logarithmic kernel: applications," IMA Journal of Numerical Analysis, vol. 8, no. 1, pp. 123-140, 1988.

[9] I. G. Graham, "Galerkin methods for second kind integral equations with singularities," Mathematics of Computation, vol. 39, no. 160, pp. 519-533, 1982.

[10] K. Orav-Puurand, A. Pedas, and G. Vainikko, "Nyström type methods for Fredholm integral equations with weak singularities," Journal of Computational and Applied Mathematics, vol. 234, no. 9, pp. 2848-2858, 2010.

[11] J. Huang, T. Lü, and Z. C. Li, "Mechanical quadrature methods and their splitting extrapolations for boundary integral equations of first kind on open arcs," Applied Numerical Mathematics, vol. 59, no. 12, pp. 2908-2922, 2009.

[12] Z. Rui, H. Jin, and L. Tao, "Mechanical quadrature methods and their splitting extrapolations for solving boundary integral equations of axisymmetric Laplace mixed boundary value problems," Engineering Analysis with Boundary Elements, vol. 30, no. 5, pp. 391-398, 2006.

[13] I. S. Gradshteyn and I. M. Ryzhik, Table of Integrals, Series, and Products, Academic Press, San Diego, Calif, USA, 7th edition, 2007.

[14] M. Fogiel, Handbook of Mathematical, Scientific, and Engineering, Research and Education Association, Piscataway, NJ, USA, 1994. 


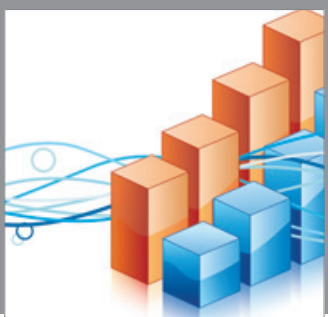

Advances in

Operations Research

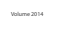

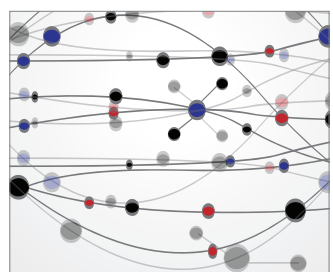

\section{The Scientific} World Journal
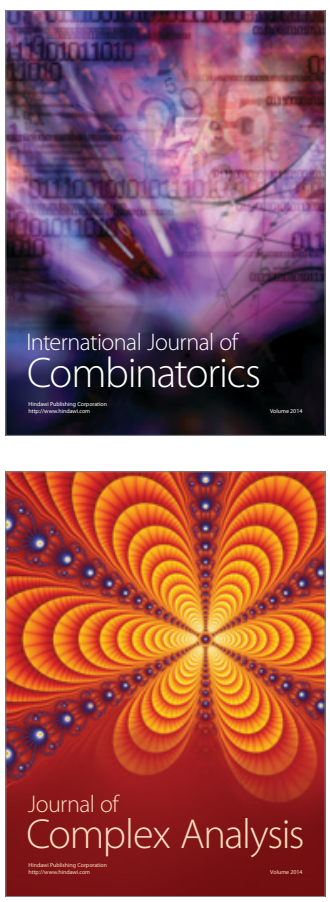

International Journal of

Mathematics and

Mathematical

Sciences
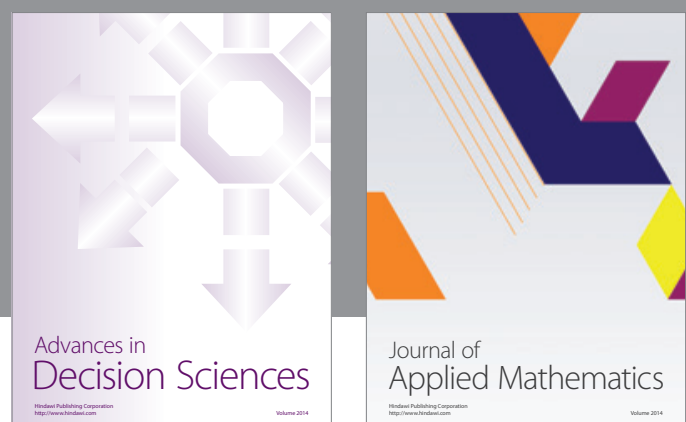

Journal of

Applied Mathematics
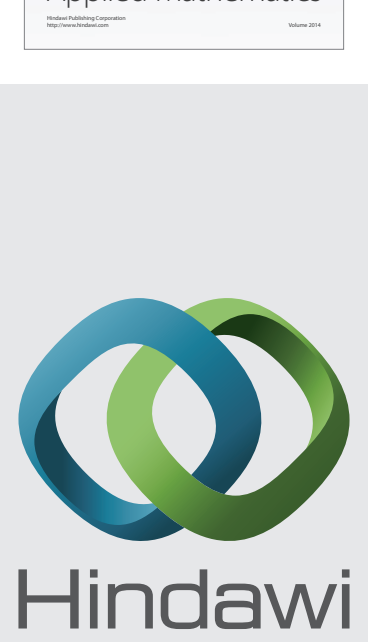

Submit your manuscripts at http://www.hindawi.com
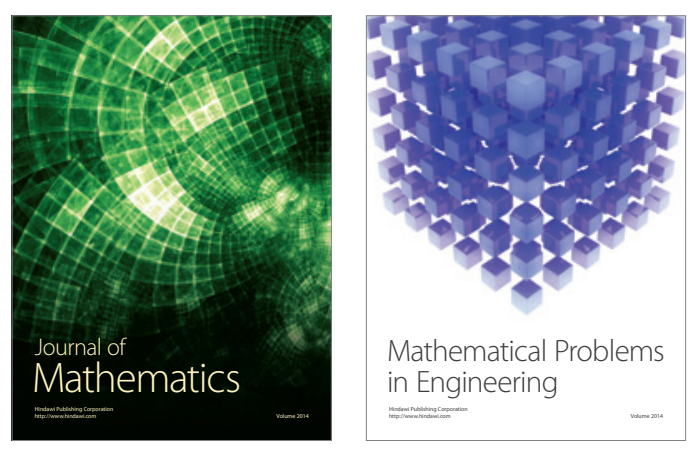

Mathematical Problems in Engineering
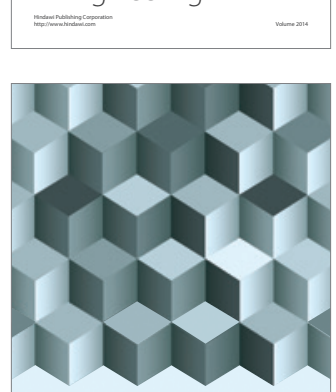

Journal of

Function Spaces
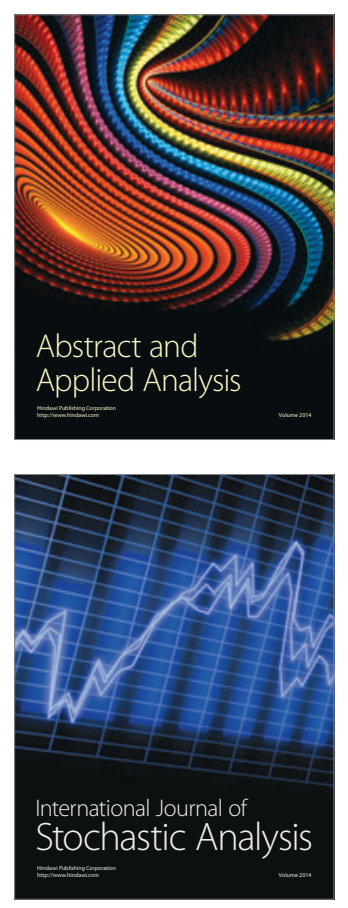

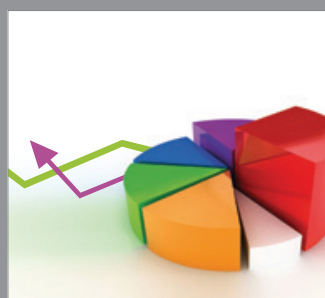

ournal of

Probability and Statistics

Promensencen
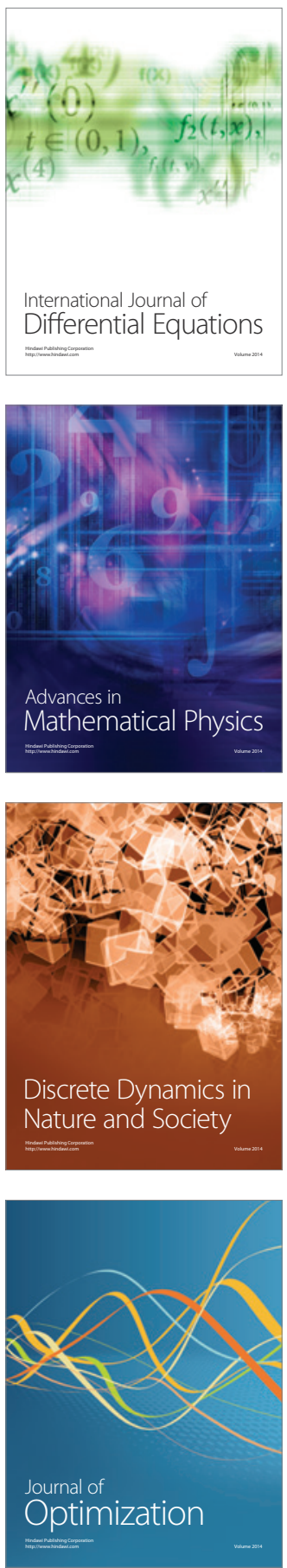